\title{
Determining the nonperturbative Collins-Soper kernel from lattice QCD
}

\author{
Markus A. Ebert, ${ }^{*}$ Iain W. Stewart, ${ }^{\dagger}$ and Yong Zhao \\ Center for Theoretical Physics, Massachusetts Institute of Technology, \\ Cambridge, Massachusetts 02139, USA
}

(Received 12 November 2018; published 19 February 2019)

\begin{abstract}
At small transverse momentum $q_{T}$, transverse-momentum dependent parton distribution functions (TMDPDFs) arise as genuinely nonperturbative objects that describe Drell-Yan like processes in hadron collisions as well as semi-inclusive deep-inelastic scattering. TMDPDFs naturally depend on the hadron momentum, and the associated evolution is determined by the Collins-Soper equation. For $q_{T} \sim \Lambda_{\mathrm{QCD}}$ the corresponding evolution kernel (or anomalous dimension) is nonperturbative and must be determined as an independent ingredient in order to relate TMDPDFs at different scales. We propose a method to extract this kernel using lattice QCD and the large-momentum effective theory, where the physical TMD correlation involving light-like paths is approximated by a quasi-TMDPDF, defined using equal-time correlation functions with a large-momentum hadron state. The kernel is determined from a ratio of quasi-TMDPDFs extracted at different hadron momenta.
\end{abstract}

DOI: 10.1103/PhysRevD.99.034505

\section{INTRODUCTION}

In the past decades, advances in theory and experiment have made it possible to explore the structure of the proton beyond the simplest longitudinal momentum distributions. Key observables are transverse momentum distributions (TMDs), which measure the intrinsic transverse momentum $q_{T}$ of partons in the proton, as well as describing the probability to produce particles at larger $q_{T}$ in high energy collisions. These TMDs are probed directly by experiments on Drell-Yan, semi-inclusive deep inelastic scattering (SIDIS), and other processes. Recently, progress has been made in determining transverse-momentum dependent parton distribution functions (TMDPDFs) by using lattice QCD [1-6] to study equal-time correlators. Such correlators are a key ingredient in the large-momentum effective theory (LaMET) [7,8], where one computes a lightcone correlator using an equal-time correlator in a boosted proton state. For TMDPDFs the first theoretical studies in LaMET have been carried out in Refs. [9-11].

The TMDPDF $f_{i}^{\mathrm{TMD}}\left(x, \vec{b}_{T}, \mu, \zeta\right)$ for a parton of flavor $i$ depends on $x$, the fraction of the hadron momentum carried by the struck parton, $\vec{b}_{T}$, the Fourier-conjugate of the

\footnotetext{
*ebert@mit.edu

iains@mit.edu

¥yzhaoqcd@mit.edu
}

Published by the American Physical Society under the terms of the Creative Commons Attribution 4.0 International license. Further distribution of this work must maintain attribution to the author(s) and the published article's title, journal citation, and DOI. Funded by SCOAP ${ }^{3}$. transverse momentum $\vec{q}_{T}$, and on a virtuality scale $\mu$. In addition, it depends on a scale $\zeta$, which is related to the momentum of the hadron or equivalently the hard scale of the scattering process. Measuring nonperturbative TMDPDFs, whether from experiment or lattice, thus requires to specify the scales $\left(\mu_{0}, \zeta_{0}\right)$ where the TMDPDF is extracted. For instance, lattice calculations are restricted to $\mu_{0}^{2} \sim \zeta_{0} \sim \mathcal{O}\left(4 \mathrm{GeV}^{2}\right)$ due to finite lattice spacing, while for example the application to Drell-Yan production uses $\mu^{2} \sim \zeta \sim m_{Z}^{2} \approx(91 \mathrm{GeV})^{2}$. The TMDPDFs thus need be evolved from $\left(\mu_{0}, \zeta_{0}\right)$ to the phenomenologically relevant scales $(\mu, \zeta)$, using

$$
\begin{aligned}
f_{i}^{\mathrm{TMD}}\left(x, \vec{b}_{T}, \mu, \zeta\right)= & f_{i}^{\mathrm{TMD}}\left(x, \vec{b}_{T}, \mu_{0}, \zeta_{0}\right) \\
& \times \exp \left[\int_{\mu_{0}}^{\mu} \frac{\mathrm{d} \mu^{\prime}}{\mu^{\prime}} \gamma_{\mu}^{i}\left(\mu^{\prime}, \zeta_{0}\right)\right] \\
& \times \exp \left[\frac{1}{2} \gamma_{\zeta}^{i}\left(\mu, b_{T}\right) \ln \frac{\zeta}{\zeta_{0}}\right] .
\end{aligned}
$$

The first exponential in Eq. (1) is the $\mu$ evolution and the second exponential is the Collins-Soper evolution in $\zeta$ [12-14] in the formulation of Ref. [15], with $\gamma_{\mu}^{i}$ and $\gamma_{\zeta}^{i}$ being the associated anomalous dimensions. Here $\gamma_{\zeta}^{i}$ is the Collins-Soper kernel, often denoted by $\tilde{K}$.

The $\mu$ evolution in Eq. (1) is perturbative as long as both $\mu_{0}, \mu \gg \Lambda_{\mathrm{QCD}}$, analogous to the perturbative DGLAP evolution for collinear PDFs. In contrast, the CollinsSoper kernel involves the $b_{T}$-dependent anomalous dimension $\gamma_{\zeta}^{i}\left(\mu, b_{T}\right)$, which becomes nonperturbative in the region $b_{T}^{-1} \sim q_{T} \sim \Lambda_{\mathrm{QCD}}$, even if $\mu \gg \Lambda_{\mathrm{QCD}}$. Relating 
the nonperturbative TMDPDF extracted at some reference scales $\left(\mu_{0}, \zeta_{0}\right)$ to the phenomenologically relevant scales $(\mu, \zeta)$ thus crucially relies on the nonperturbative knowledge of the Collins-Soper kernel.

Due to the simple form of Eq. (1), the Collins-Soper evolution can be factored out by taking the ratio of two TMDPDFs extracted at different values $\zeta_{1} \neq \zeta_{2}$,

$$
\gamma_{\zeta}^{i}\left(\mu, b_{T}\right)=\frac{2}{\ln \left(\zeta_{1} / \zeta_{2}\right)} \ln \frac{f_{i}^{\mathrm{TMD}}\left(x, \vec{b}_{T}, \mu, \zeta_{1}\right)}{f_{i}^{\mathrm{TMD}}\left(x, \vec{b}_{T}, \mu, \zeta_{2}\right)} .
$$

One option is to extract $\gamma_{\zeta}^{i}\left(\mu, b_{T}\right)$ experimentally. For example, one can use Drell-Yan production at small $q_{T} \ll Q$, where $Q$ is the invariant mass of the Drell-Yan pair, and use different values of $Q$ to obtain $f_{i=q}^{\mathrm{TMD}}\left(x, \vec{b}_{T}, \mu, \zeta\right)$ at different $\zeta$ values. This can be done using results from global fits, see e.g., Refs. [16,17]. Experimentally, the TMDPDF is extracted as a function of $\vec{q}_{T}$, which makes it challenging to use Eq. (2) since it requires the Fourier transformation into $\vec{b}_{T}$ space. Interestingly, Eq. (2) is independent of the momentum fraction $x$ and choice of $\zeta_{1,2}$, which is useful to assess associated systematics and to validate the applicability of TMD factorization.

In this paper, we propose a first-principle method of determining the nonperturbative $\gamma_{\zeta}^{q}$ using lattice QCD. A potential benefit is that one has, in principle, more control over the systematics in the calculation. The TMDPDFs in Eq. (2) are not directly computable on the lattice because they involve time dependent operators with Wilson lines on (or close to) the light cone. We therefore consider the LaMET approach for calculating the ratio in Eq. (2) from lattice QCD, which will involve additional perturbative matching corrections. Our proposed formula for $\gamma_{\zeta}^{q}\left(\mu, b_{T}\right)$ is analogous to Eq. (2) and is given in Sec. IV.

Below in Sec. II, we briefly review the definition of TMDPDFs in the context of TMD factorization for protonproton collisions. In Sec. III, we present the construction of quasi-TMDPDFs using LaMET that are computable on lattice, and briefly address issues arising from the presence of both collinear and soft matrix elements. The strategy to extract $\gamma_{\zeta}^{q}$ from lattice is presented in Sec. IV. We conclude in Sec. V.

\section{TMD FACTORIZATION}

TMD factorization was originally derived by Collins, Soper and Sterman (CSS) in Refs. [12-14] and extended by Collins in Ref. [15]. The cancellation of potentially factorization-violating Glauber contributions has been shown in Refs. [15,18-21]. The factorization has also been considered in the framework of soft-collinear effective theory (SCET) [22-25] by various authors [26-32], see, e.g., Ref. [33] for a detailed discussion of the different approaches.
We consider the production of a color-singlet final state $F$ with invariant mass $Q$, rapidity $Y$ and small transverse momentum $q_{T}=\left|\vec{q}_{T}\right| \ll Q$ in the scattering of two energetic protons moving close to the $n^{\mu}=$ $(1,0,0,1)$ and $\bar{n}^{\mu}=(1,0,0,-1)$ directions with a center of mass energy $E_{\mathrm{cm}}$. In the limit $q_{T} \ll Q$ the cross section can be factorized as

$$
\begin{aligned}
\frac{\mathrm{d} \sigma}{\mathrm{d} Q \mathrm{~d} Y \mathrm{~d}^{2} \vec{q}_{T}}= & \sum_{i, j} H_{i j}(Q, \mu) \int \mathrm{d}^{2} \vec{b}_{T} e^{\mathrm{i} \vec{b}_{T} \cdot \vec{q}_{T}} \\
& \times f_{i}^{\mathrm{TMD}}\left(x_{a}, \vec{b}_{T}, \mu, \zeta_{a}\right) f_{j}^{\mathrm{TMD}}\left(x_{b}, \vec{b}_{T}, \mu, \zeta_{b}\right),
\end{aligned}
$$

which holds up to power corrections of relative order $q_{T}^{2} / Q^{2}$ and $\Lambda_{\mathrm{QCD}}^{2} / Q^{2}$, but remains valid in the nonperturbative regime $q_{T} \sim \Lambda_{\mathrm{QCD}}$. Here, $H_{i j}$ is the hard function describing virtual corrections to the underlying hard process $i j \rightarrow F$, where $i, j$ are the parton flavors (for gluoninduced process, $i j=g g$, the hard function and the TMDPDFs carry helicity indices, which are suppressed here), $x_{a, b}=Q e^{ \pm Y} / E_{\mathrm{cm}}$ are the longitudinal momentum fractions carried by the struck partons, and $f^{\mathrm{TMD}}$ are the TMDPDFs in Fourier space. (Our $H$ and $f^{\mathrm{TMD}}$ s agree with the definitions used in Refs. [15,26,29,32,34-38], but differ from those of Refs. [12-14,39], see Refs. [33,40] for relations.) Note that these physical TMDs involve staple Wilson line paths, and hence differ from the straight line paths considered in Refs. [1,2,41]. A similar result is obtained for SIDIS, $e p \rightarrow h+X$, where the transverse momentum of the hadron $h$ is measured by a TMD fragmentation function rather than a TMDPDF, which obeys the same Collins-Soper equation [15].

An important feature of TMDs is that bare matrix elements not only suffer from UV divergences regulated by $\epsilon$ (for example in dimensional regularization with $d=4-2 \epsilon$ ), but also from rapidity divergences (also known as lightcone divergences) which require another regulator $[12,26,29,32,42-44]$ that we will generically denote by $\tau$. Many such regulators have been suggested in the literature $[12,15,29,32,35,37,39,44-47]$. As a consequence, TMDPDFs depend on both the renormalization scale $\mu$ and the parameter $\zeta$. In Eq. (3) $\mu$ formally cancels between the $H$ and $f^{\mathrm{TMD}}$ functions, but in practice is chosen as $\mu \sim Q$ to avoid large logarithms $\ln (Q / \mu)$ in the hard function. The values for $\zeta_{a}$ and $\zeta_{b}$ are not fixed individually, but their product is fixed to $\zeta_{a} \zeta_{b}=Q^{4}$. This forces one to evaluate the TMDPDFs at $\mu^{2} \sim \zeta \sim Q^{2}$, and requires the use of Eq. (1) to evolve them from some other scales like $\left(\mu_{0}^{2}, \zeta_{0}\right) \sim \mathcal{O}\left(4 \mathrm{GeV}^{2}\right)$ where they are nonperturbatively determined (or parametrized).

A definition of the quark TMDPDF consistent with Refs. $[15,29,32]$ can be given by 


$$
\begin{aligned}
f_{q}^{\mathrm{TMD}}\left(x, \vec{b}_{T}, \mu, \zeta\right)= & \lim _{\substack{\epsilon \rightarrow 0 \\
\tau \rightarrow 0}} Z_{\mathrm{uv}}(\mu, \zeta, \epsilon) B_{q}\left(x, \vec{b}_{T}, \epsilon, \tau, \zeta\right) \\
& \times \Delta_{S}^{q}\left(b_{T}, \epsilon, \tau\right) .
\end{aligned}
$$

Here $Z_{\mathrm{uv}}$ is the ultraviolet (UV) renormalization factor, and we refer to $B_{q}$ and $\Delta_{S}^{q}$ as the bare beam function (where we follow the naming scheme of Ref. [48]) and soft factor, respectively, to distinguish them from the TMDPDF $f_{q}^{\mathrm{TMD}}$. References $[15,29,32]$ use different definitions for $\tau, B_{q}$, and $\Delta_{S}^{q}$ but all choices yield the same $f_{q}^{\mathrm{TMD}}$. As $\tau \rightarrow 0$ only the combination $1 / \tau-\ln \sqrt{\zeta}$ shows up in the bare function $B_{q}$. Importantly, $1 / \tau$ divergences cancel out in Eq. (4) yielding a well-defined TMDPDF. A remnant of this cancellation is the appearance of $\zeta$ in $f_{q}^{\mathrm{TMD}}$. In Eq. (3) we have

$$
\zeta_{a}=x_{a}^{2}\left(P_{a}^{-}\right)^{2} e^{-2 y_{n}}, \quad \zeta_{b}=x_{b}^{2}\left(P_{b}^{+}\right)^{2} e^{2 y_{n}},
$$

where $P_{a, b}$ are the hadron momenta and $y_{n}$ is an arbitrary parameter controlling the split of soft radiation into the TMDPDFs (where the precise specification of $y_{n}$ differs in Refs. $[15,29,32])$. Their product is always fixed to

$$
\zeta_{a} \zeta_{b}=Q^{4}=\left(x_{a} x_{b} E_{\mathrm{cm}}^{2}\right)^{2} .
$$

Eqs. (5) and (6) involve the momentum fractions $x_{a}$ and $x_{b}$, and thus are specified in momentum space.

A well-known example of Eq. (4) is Collins' regulator [15] where Wilson lines are taken off the lightcone, and the soft factor is defined as

$$
\begin{aligned}
\Delta_{S}^{q}\left(b_{T}, \epsilon, \tau\right) & =\lim _{y_{A} \rightarrow \infty} \sqrt{\frac{S^{y_{A}, y_{n}}}{S^{y_{A}, y_{B}} S^{y_{n}, y_{B}}}} \\
& =\frac{1}{\sqrt{S_{C}^{q}\left(b_{T}, \epsilon, 2 y_{n}-2 y_{B}\right)}},
\end{aligned}
$$

(see Ref. [49] for the last equality). Here, $S^{y_{a}, y_{b}}$ denotes a soft matrix element with Wilson lines parametrized by the rapidities $y_{a}$ and $y_{b}$, and $y_{A}$ and $y_{B}$ are the rapidities of the Wilson lines entering $B_{q}$ for the $n$-collinear proton and $\bar{n}$ collinear proton, respectively. The regulator is given by $\tau=$ $1 /\left(y_{B}-y_{n}\right)$ with $y_{B} \rightarrow-\infty$. For Collins' beam function we have

$$
B_{q}\left(x, \vec{b}_{T}, \epsilon, \tau, \zeta\right)=B_{q}^{\mathrm{C}}\left(x, \vec{b}_{T}, \epsilon, y_{P}-y_{B}\right),
$$

which only depends on the rapidity difference $y_{P}-y_{B}$ since $1 / \tau-\ln \sqrt{\zeta}=y_{B}-y_{P}-\ln \left(x m_{P}\right)$, where $m_{P}$ and $y_{P}$ are the proton mass and rapidity, and $P^{-}=m_{P} e^{y_{P}}$ is the proton momentum.

In the EIS scheme $[29,30]$ one regulates eikonal propagators by basically shifting $1 /\left(k^{ \pm}+\mathrm{i} 0\right) \rightarrow 1 /\left(k^{ \pm}+\mathrm{i} \delta^{ \pm}\right)$. In this scheme there is a soft function $S$ and two zero-bin subtraction [50] functions $S_{0}$ which avoid double counting between the soft function $S$ and the beam function $B$. The $S$ and $S_{0}$ appear together as a multiplicative factor of $S /\left(S_{0} S_{0}\right)=1 / S=1 /(\sqrt{S} \sqrt{S})$. With the $\delta^{ \pm}$regulators one therefore defines the soft factor appearing in the TMD as

$$
\Delta_{S}^{q}\left(b_{T}, \epsilon, \tau\right)=\frac{1}{\sqrt{S_{\mathrm{EIS}}^{q}\left(b_{T}, \epsilon, \delta^{-} e^{-y_{n}}\right)}} .
$$

Here the regulator is $1 / \tau=\ln \left(\delta^{-} e^{-y_{n}}\right)$. In this scheme

$$
B_{q}\left(x, \vec{b}_{T}, \epsilon, \tau, \zeta\right)=B_{q}^{\mathrm{EIS}}\left(x, \vec{b}_{T}, \epsilon, \delta^{-} /\left(x P^{-}\right)\right),
$$

where $B_{q}^{\mathrm{EIS}} \equiv J_{n}$ in the notation of Ref. [30] and $1 / \tau-\ln \sqrt{\zeta}=\ln \left[\delta^{-} /\left(x P^{-}\right)\right]$.

Finally, in the $\eta$-regulator and scheme of Chiu-JainNeill-Rothstein (CJNR) in Ref. [32] the Wilson lines in the proton and vacuum matrix elements are regulated with factors of $\left|2 k^{z}\right|^{-\eta}$, where $k$ is the momentum of gluons emitted from the Wilson line, and we have $\tau=\eta$. In this scheme the zero-bin subtraction functions vanish, so $S /\left(S_{0} S_{0}\right)=S=\sqrt{S} \sqrt{S}$. Therefore the soft factor is

$$
\Delta_{S}^{q}\left(b_{T}, \epsilon, \tau\right)=\sqrt{S_{\mathrm{CJNR}}^{q}\left(b_{T}, \epsilon, \eta\right)} .
$$

In this approach the regulator is chosen to act symmetrically on the two proton matrix elements, so $y_{n}=0$, $\zeta=\left(x P^{-}\right)^{2}$, and the bare beam function is

$$
B_{q}\left(x, \vec{b}_{T}, \epsilon, \tau, \zeta\right)=B_{q}^{\mathrm{CJNR}}\left(x, \vec{b}_{T}, \epsilon, \eta,\left(x P^{-}\right)^{2}\right) .
$$

The exponential regulator defined in Ref. [37], and used for the three-loop perturbative computation of $\gamma_{\zeta}$ in Ref. [51], has a similar functional dependence in $S$ and $B_{q}$ as the $\eta$-regulator (though the zero-bin subtraction functions $S_{0}$ do not vanish in this case). So far this scheme has only been used for perturbative calculations, but it also belongs in the set of TMDPDF definitions governed by Eq. (4).

In general $B_{q}$ involves a matrix element with an energetic hadron moving along the $n$ direction, and encodes the effect of collinear radiation associated to the hadron $h$ of momentum $P$, and $S^{q}\left(b_{T}, \epsilon, \tau\right)$ is a bare soft vacuum matrix element which encodes the effect of soft interactions between the incoming partons. Their precise definitions depend on the regulator $\tau$, which we leave implicit for simplicity, 


$$
\begin{aligned}
& B_{q}\left(x, \vec{b}_{T}, \epsilon, \tau, \zeta\right) \\
& =\int \frac{\mathrm{d} b^{+}}{4 \pi} e^{-\mathrm{i} \frac{1}{2} b^{+}\left(x P^{-}\right)}\langle h(P)| \\
& \quad \times\left[\bar{q}\left(b^{\mu}\right) W\left(b^{\mu}\right) \frac{\gamma^{-}}{2} W_{T}\left(-\infty \bar{n} ; \vec{b}_{T}, \overrightarrow{0}_{T}\right) W^{\dagger}(0) q(0)\right]_{\tau}|h(P)\rangle,
\end{aligned}
$$

$$
\begin{aligned}
S^{q}\left(b_{T}, \epsilon, \tau\right) & \\
= & \frac{1}{N_{c}}\langle 0| \operatorname{Tr}\left[S_{n}^{\dagger}\left(\vec{b}_{T}\right) S_{\bar{n}}\left(\vec{b}_{T}\right) S_{T}\left(-\infty \bar{n} ; \vec{b}_{T}, \overrightarrow{0}_{T}\right)\right. \\
& \left.\times S_{\bar{n}}^{\dagger}\left(\overrightarrow{0}_{T}\right) S_{n}\left(\overrightarrow{0}_{T}\right) S_{T}^{\dagger}\left(-\infty n ; \vec{b}_{T}, \overrightarrow{0}_{T}\right)\right]_{\tau}|0\rangle .
\end{aligned}
$$

We use the lightcone coordinates $b^{ \pm}=b^{0} \mp b^{z}$, such that $b^{\mu}=b^{+} \bar{n}^{\mu} / 2+b_{T}^{\mu}$. The Wilson lines appearing in Eqs. (13) and (14) are defined as path-ordered exponentials,

$$
\begin{aligned}
W\left(x^{\mu}\right) & =P \exp \left[-\mathrm{i} g \int_{-\infty}^{0} \mathrm{~d} s \bar{n} \cdot \mathcal{A}\left(x^{\mu}+s \bar{n}^{\mu}\right)\right], \\
S_{n}\left(x^{\mu}\right) & =P \exp \left[-\mathrm{i} g \int_{-\infty}^{0} \mathrm{~d} s n \cdot \mathcal{A}\left(x^{\mu}+s n^{\mu}\right)\right], \\
W_{T}\left(x^{\mu} ; \vec{b}_{T}, \overrightarrow{0}_{T}\right) & =P \exp \left[\mathrm{i} g \int_{\overrightarrow{0}_{T}}^{\vec{b}_{T}} \mathrm{~d} \vec{s}_{T} \cdot \overrightarrow{\mathcal{A}}_{T}\left(x^{\mu}+s_{T}^{\mu}\right)\right] \\
& =S_{T}\left(x^{\mu} ; \vec{b}_{T}, \overrightarrow{0}_{T}\right) .
\end{aligned}
$$

For clarity, we use a different notation for soft Wilson lines $S$ and collinear Wilson lines $W$. The Wilson line paths in Eqs. (13) and (14) are illustrated in Fig. 1.

The dependence of the TMDPDF on the scales $\mu$ and $\zeta$ is governed by the renormalization group equations

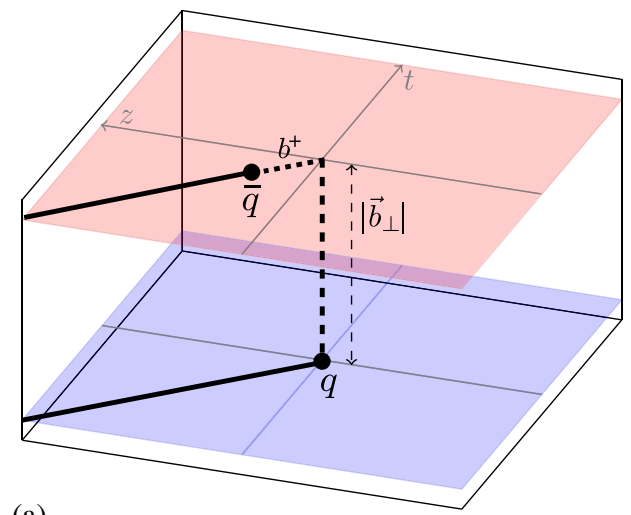

(a)

$$
\begin{aligned}
\mu \frac{\mathrm{d}}{\mathrm{d} \mu} f_{i}^{\mathrm{TMD}}\left(x, \vec{b}_{T}, \mu, \zeta\right) & =\gamma_{\mu}^{i}(\mu, \zeta) f_{i}^{\mathrm{TMD}}\left(x, \vec{b}_{T}, \mu, \zeta\right), \\
\zeta \frac{\mathrm{d}}{\mathrm{d} \zeta} f_{i}^{\mathrm{TMD}}\left(x, \vec{b}_{T}, \mu, \zeta\right) & =\frac{1}{2} \gamma_{\zeta}^{i}\left(\mu, b_{T}\right) f_{i}^{\mathrm{TMD}}\left(x, \vec{b}_{T}, \mu, \zeta\right), \\
\mu \frac{\mathrm{d}}{\mathrm{d} \mu} \gamma_{\zeta}^{i}\left(\mu, b_{T}\right) & =2 \zeta \frac{\mathrm{d}}{\mathrm{d} \zeta} \gamma_{\mu}^{i}(\mu, \zeta) \\
& =-2 \Gamma_{\text {cusp }}^{i}\left[\alpha_{s}(\mu)\right],
\end{aligned}
$$

where the second equation is the Collins-Soper equation $[12,13]$. It can also be written as a convolution in momentum space [13], where its solution is more complicated [52]. The combined solution to Eq. (16) yields the evolution in Eq. (1), where we have chosen a specific path in the $(\mu, \zeta)$ plane, but one is free to choose any path connecting $\left(\mu_{0}, \zeta_{0}\right) \rightarrow(\mu, \zeta)$ since the last equation in Eq. (16) ensures path independence (see also Ref. [53]).

The subscripts on the anomalous dimensions $\gamma_{\mu}^{i}$ and $\gamma_{\zeta}^{i}$ label the scale evolution they govern. Their all-order forms are given by

$$
\begin{aligned}
\gamma_{\mu}^{i}(\mu, \zeta) & =\Gamma_{\text {cusp }}^{i}\left[\alpha_{s}(\mu)\right] \ln \frac{\mu^{2}}{\zeta}+\gamma_{\mu}^{i}\left[\alpha_{s}(\mu)\right], \\
\gamma_{\zeta}^{i}\left(\mu, b_{T}\right) & =-2 \int_{1 / b_{T}}^{\mu} \frac{\mathrm{d} \mu^{\prime}}{\mu^{\prime}} \Gamma_{\text {cusp }}^{i}\left[\alpha_{s}\left(\mu^{\prime}\right)\right]+\gamma_{\zeta}^{i}\left[\alpha_{s}\left(1 / b_{T}\right)\right] .
\end{aligned}
$$

They both have a piece governed by the cusp anomalous dimension $\Gamma_{\text {cusp }}^{i}\left[\alpha_{s}\right]$, and a noncusp piece $\gamma^{i}\left[\alpha_{s}\right]$. Both anomalous dimension differ for quarks, $i=q$, and gluons, $i=g$, but are independent of the choice of hadron state and the light quark flavor in the operator in Eq. (13) (for $b$-quarks see Ref. [54]). For $\gamma_{\zeta}^{i}$ this follows because

$\gamma_{\zeta}^{i}=2 \frac{\mathrm{d} \ln f_{i}^{\mathrm{TMD}}}{\mathrm{d} \ln \zeta}=2 \frac{\mathrm{d} \ln B_{i}}{\mathrm{~d} \ln \zeta}=-\frac{\mathrm{d} \ln B_{i}}{\mathrm{~d}(1 / \tau)}=\frac{\mathrm{d} \ln \Delta_{S}^{i}}{\mathrm{~d}(1 / \tau)}$,

and $\Delta_{S}^{i}$ does not depend on the hadron state.

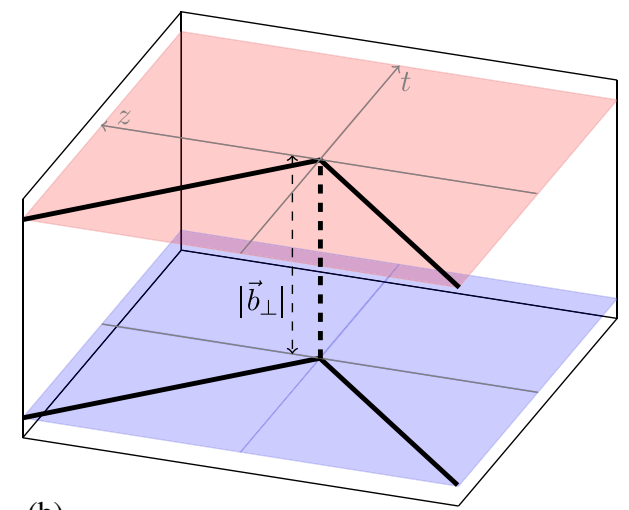

(b)

FIG. 1. Illustration of the Wilson line structure of (a) the $n$-collinear beam function $B_{q}$ and (b) the soft function $S^{q}$, defined in Eqs. (13) and (14). The Wilson lines (solid) extend to infinity in the directions indicated and are joined there by transverse Wilson lines. The $\tau$ dependence that regulates singularities from these Wilson lines is not shown. Adapted from Ref. [37]. 
Equation (17) clearly shows that if $b_{T} \sim \Lambda_{\mathrm{QCD}}^{-1}$ then $\gamma_{\zeta}^{i}\left(\mu, b_{T}\right)$ has an intrinsically nonperturbative component. Once $\gamma_{\zeta}^{i}\left(\mu, b_{T}\right)$ is determined at a scale $\mu_{0} \gg \Lambda_{\mathrm{QCD}}$, it can be perturbatively determined at any scale $\mu \gg \Lambda_{\mathrm{QCD}}$ via

$$
\gamma_{\zeta}^{i}\left(\mu, b_{T}\right)=\gamma_{\zeta}^{i}\left(\mu_{0}, b_{T}\right)-2 \int_{\mu_{0}}^{\mu} \frac{\mathrm{d} \mu^{\prime}}{\mu^{\prime}} \Gamma_{\text {cusp }}^{i}\left[\alpha_{s}\left(\mu^{\prime}\right)\right] .
$$

The result for $\gamma_{\zeta}^{i}\left(\mu_{0}, b_{T}\right)$ is known to 3-loop order for perturbative $b_{T}[37,51,55]$. The focus of this paper is to determine $\gamma_{\zeta}^{i}\left(\mu_{0}, b_{T}\right)$ nonperturbatively, which can be used for the evolution even when $b_{T} \sim \Lambda_{\mathrm{QCD}}^{-1}$.

\section{TMDPDFS ON THE LATTICE}

While lattice QCD provides a practical tool for firstprinciple calculations of nonperturbative quantities, it has long been challenging to compute lightcone correlators on the Euclidean lattice due to their real-time dependence. LaMET has been proposed to overcome this hurdle by relating the light-cone correlator to an equal-time correlator in a highly boosted hadron state $[7,8]$. The latter can be calculated on lattice, and can then be matched onto the corresponding lightcone matrix elements through a systematic expansion in the hadron momentum $P^{z}$, as proven in Refs. [56-58].

Using the LaMET approach, it has been suggested to calculate TMDPDFs in a similar fashion [9-11]. An earlier and related approach used in lattice calculations is to exploit Lorentz invariance for the spacelike correlator $[2,3]$. To begin with, we define a bare quasi beam function in position space as

$$
\begin{aligned}
\tilde{B}_{q}\left(b^{z}, \vec{b}_{T}, a, L, P^{z}\right) \\
=\langle h(P)| \bar{q}\left(b^{\mu}\right) W_{\hat{z}}\left(b^{\mu} ; L-b^{z}\right) \\
\quad \times \frac{\Gamma}{2} W_{T}\left(L \hat{z} ; \vec{b}_{T}, \overrightarrow{0}_{T}\right) W_{\hat{z}}^{\dagger}(0 ; L) q(0)|h(P)\rangle,
\end{aligned}
$$

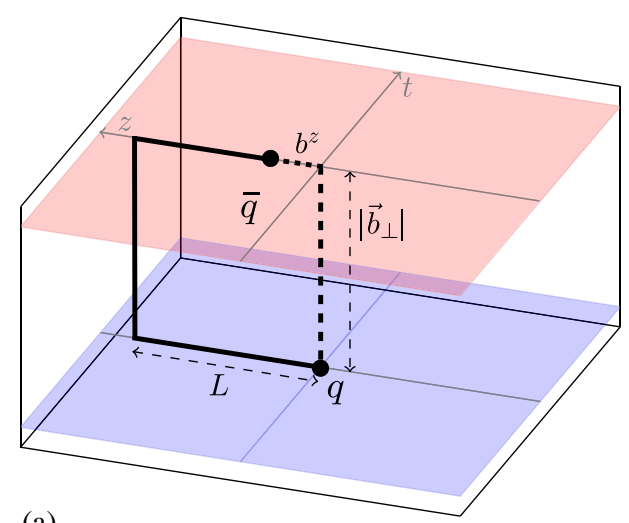

(a) where $b^{\mu}=\left(0, \vec{b}_{T}, b^{z}\right)$ and $a$ denotes the lattice spacing which acts as an UV regulator, but for simplicity we stick to continuum notation for the fields. In Eq. (20), one has either $\Gamma=\gamma^{0}$ or $\Gamma=\gamma^{z}$, as both can be boosted onto $\gamma^{-}$. Just like for the quasi-PDF the choice of $\Gamma=\gamma^{0}$ might be preferred on the lattice to avoid operator mixing [59-61].

Compared to Eq. (13), in Eq. (20) the lightcone Wilson lines are replaced by purely spatial Wilson lines of length $L$ because of the finite lattice size,

$$
W_{\hat{z}}\left(x^{\mu} ; L\right)=P \exp \left[\mathrm{i} g \int_{L}^{0} \mathrm{~d} s \mathcal{A}^{z}\left(x^{\mu}+s \hat{z}\right)\right] .
$$

This also regulates the analog of rapidity divergences in $\tilde{B}_{q}$, as has been shown explicitly in Refs. [10,11], and thus $L$, in part, takes the role of the rapidity regulator $\tau$ in Eq. (13). The inclusion of transverse gauge links ensures gauge invariance [62-65]. The resulting Wilson line structure of Eq. (20) is illustrated in Fig. 2(a). The same correlator in Eq. (20) has been used in the lattice calculation of ratios of TMDPDFs with $b^{z}=0$ in Refs. [3-6].

Under a Lorentz boost along the $z$ direction with velocity $v \rightarrow 1$ and boost parameter $\gamma=1 / \sqrt{1-v^{2}}$, the spatial separation behaves as $\hat{z}=(0,0,0,1) \rightarrow \gamma(-v, 0,0,1) \approx-\gamma \bar{n}$. This boost behavior is illustrated in Fig. 2(b). It is easy to see that by applying this relation to Eq. (20), one recovers Eq. (13) in the limit $v \rightarrow 1$. This suggests that a matching between Eqs. (13) and (20) could be possible, similar to the collinear PDF, up to possible issues from regulating rapidity divergences, as such regulators necessarily break boost invariance, see Ref. [11].

For the soft matrix element defined in Eq. (14), a simple quasiconstruction is not possible, since the Wilson lines involve both lightcone directions $n$ and $\bar{n}$, which would require opposite boosts to be recovered from spatial Wilson lines along the $\pm \hat{z}$ directions. A detailed study of this issue is given in Ref. [11]. Due to this issue, we will simply introduce an intrinsically nonperturbative quantity

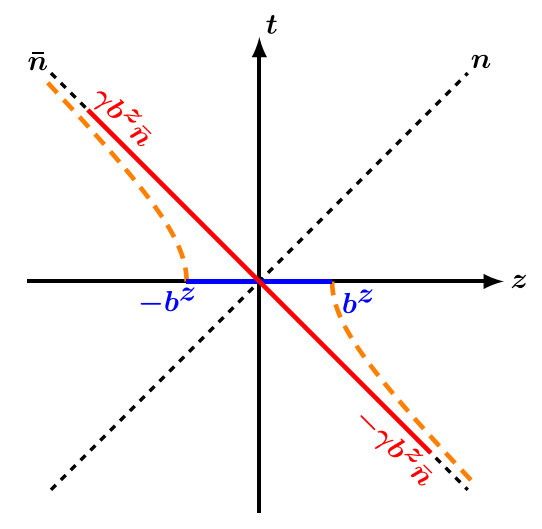

(b)

FIG. 2. (a): Illustration of the Wilson line structure of the quasi beam function $\tilde{B}_{q}$ in Eq. (20). (b): Behavior of a longitudinal separation $b^{z}$ (blue solid) under a Lorentz boost along the $z$ direction (orange dotted), and its approximate limit $-\gamma b^{z} \bar{n}$. 
$\tilde{\Delta}_{S}^{q}\left(b_{T}, a, L\right)$ to describe the missing infrared (IR) physics and dependence on $b_{T}$.

We define the quasi-TMDPDF in the $\overline{\mathrm{MS}}$ scheme analogous to Eq. (4) as

$\tilde{f}_{q}^{\mathrm{TMD}}\left(x, \vec{b}_{T}, \mu, P^{z}\right)=\int \frac{\mathrm{d} b^{z}}{2 \pi} e^{\mathrm{i} b^{z}\left(x P^{z}\right)} \tilde{f}_{q}^{\mathrm{TMD}}\left(b^{z}, \vec{b}_{T}, \mu, P^{z}\right)$,

where the $\overline{\mathrm{MS}}$ position-space quasi-TMDPDF is

$$
\begin{aligned}
\tilde{f}_{q}^{\mathrm{TMD}}\left(b^{z}, \vec{b}_{T}, \mu, P^{z}\right)= & \tilde{Z}^{\prime}\left(b^{z}, \mu, \tilde{\mu}\right) \tilde{Z}_{\mathrm{uv}}\left(b^{z}, \tilde{\mu}, a\right) \\
& \times \tilde{B}_{q}\left(b^{z}, \vec{b}_{T}, a, L, P^{z}\right) \tilde{\Delta}_{S}^{q}\left(b_{T}, a, L\right) .
\end{aligned}
$$

Here the quasi soft factor $\tilde{\Delta}_{S}^{q}$ also serves as a counterterm to cancel $L / b_{T}$ divergences in $\tilde{B}_{q}$. The $\tilde{Z}_{\text {uv }}$ carries out UV renormalization, which could be done nonperturbatively on the lattice, and $\tilde{\mu}$ denotes any renormalization scales this introduces. The conversion factor $\tilde{Z}^{\prime}$ converts the result into the $\overline{\mathrm{MS}}$ scheme at the scale $\mu$, which necessarily is perturbative. On the lattice there will be linear power divergences $\sim 1 / a$ coming from the spacelike Wilson-line self energies. The quasi soft factor $\tilde{\Delta}_{S}^{q}$ cancels the $L / a$ Wilson line self energy divergences appearing in $\tilde{B}_{q}$, which leaves only $b^{z} / a$ divergences to be canceled by $\tilde{Z}_{\mathrm{uv}}$. One can consider removing these $1 / a$ divergences with a counterterm determined from the static quark-antiquark potential as in Ref. [2], or with the RI/MOM scheme like in the quasi-PDF $[59,66]$, or with the gradient flow method [67].

In Eq. (23) we suppress the leftover $L$ dependence which vanishes in the physical limit $L \rightarrow \infty$. It would be interesting to construct a direct proof of these renormalization properties of the quasi-TMDPDF, along the lines of those for the quasi-PDF in Ref. [68,69]. Equation (23) has been explicitly verified in perturbation theory at one-loop order, where definitions of $\tilde{\Delta}_{S}^{q}$ that cancel all divergences in $L$ have also been given [10,11].

The quasi-TMDPDF defined in Eq. (22) is not a boost invariant quantity and thus explicitly depends on the hadron momentum $P^{z}$, which also plays the role of the variable $\zeta$ in the TMDPDF. We will exploit this $P^{z}$ dependence for our method to extract $\gamma_{\zeta}^{q}$. Importantly, $\tilde{\Delta}_{S}^{q}$ does not depend on $x$ or $P^{z}$. It also does not depend on the quark flavor, but differs from $\tilde{\Delta}_{S}^{g}$ for gluons. Thus, $\tilde{\Delta}_{S}^{q}$ drops out of ratios that are flavor blind, which will be a crucial ingredient to our proposed method, as one does not need to precisely define or calculate $\tilde{\Delta}_{S}^{q}$ on the lattice. Note that we convert $\tilde{f}_{q}^{\mathrm{TMD}}$ to the $\overline{\mathrm{MS}}$ scheme with $\tilde{Z}^{\prime}$ to simplify carrying out the matching onto the TMDPDF, though matching results for other schemes could be considered.

\section{A. Relating quasi-TMDPDF and TMDPDF}

For the collinear PDF, LaMET gives a perturbative matching between the quasi-PDF and PDF, and the same may be true between the quasi beam function and beam function. However, for the full TMDPDF this matching is potentially spoiled by the presence of the soft factors $\Delta_{S}^{q}$ and $\tilde{\Delta}_{S}^{q}$ that cannot be related simply through a Lorentz boost. For our purposes, we define $g_{q}^{S}\left(b_{T}, \mu\right)$ as the mismatch of the lightlike and quasi soft factors, which we allow to be nonperturbative, and we will exploit the fact that due to its soft origin it is independent of $x, P^{z}$, and quark flavor. For a flavor nonsinglet channel such as $u-d$ the relation between the $\overline{\mathrm{MS}}$ quasi-TMDPDF and TMDPDF is thus expected to take the form [11]

$$
\begin{aligned}
\tilde{f}_{\mathrm{ns}}^{\mathrm{TMD}}\left(x, \vec{b}_{T}, \mu, P^{z}\right)= & C_{\mathrm{ns}}^{\mathrm{TMD}}\left(\mu, x P^{z}\right) g_{q}^{S}\left(b_{T}, \mu\right) \\
& \times \exp \left[\frac{1}{2} \gamma_{\zeta}^{q}\left(\mu, b_{T}\right) \ln \frac{\left(2 x P^{z}\right)^{2}}{\zeta}\right] \\
& \times f_{\mathrm{ns}}^{\mathrm{TMD}}\left(x, \vec{b}_{T}, \mu, \zeta\right) .
\end{aligned}
$$

We suppress explicit corrections to Eq. (24) in $b_{T} / L$, $1 /\left(b_{T} P^{z}\right), 1 /\left(P^{z} L\right)$ and $\Lambda_{\mathrm{QCD}} / P^{z}$ arising from the finite hadron momentum $P^{z}$ and finite lattice size. Like for the quasi-PDF [70] there are also hadron mass corrections, $M_{h} / P^{z}$, which can likely be accounted for exactly. Eq. (24) has been explicitly verified at one loop in Ref. [11]. It involves a perturbative short distance coefficient $C_{\mathrm{ns}}^{\mathrm{TMD}}$, which is independent of $b_{T}$. It is multiplicative in $x$ space, which is known to hold at least to one-loop order [10,11], in contrast to the quasi-PDF, whose matching onto the PDF involves a convolution in $x$ [71].

The exponential in Eq. (24) contains the nonperturbative Collins-Soper kernel $\gamma_{\zeta}^{q}\left(\mu, b_{T}\right)$ that we are after. It guarantees that $\tilde{f}_{\mathrm{ns}}^{\mathrm{TMD}}$ is independent of $\zeta$ by balancing the Collins-Soper evolution of $f_{\mathrm{ns}}^{\mathrm{TMD}}$. This exponential is $\vec{b}_{T}$ dependent and thus can not be included in the shortdistance $C_{\mathrm{ns}}^{\mathrm{TMD}}$. The $\zeta$ dependence in this exponential is balanced by $\tilde{\zeta} \equiv\left(2 x P^{z}\right)^{2}$, which is the Collins-Soper scale of Eq. (5) away from the lightcone, corresponding with the $z$-momentum of the struck quark. For our use of Eq. (24) we are considering $\tilde{\zeta}$ and $\zeta$ to be independent variables. This can be equivalently thought of as having different values of $P^{z}$ in the quasi-TMDPDF $\tilde{f}_{\mathrm{ns}}^{\mathrm{TMD}}$ and TMDPDF $f_{\mathrm{ns}}^{\mathrm{TMD}}$, where the nonperturbative $\gamma_{\zeta}^{q}\left(\mu, b_{T}\right)$ is then needed to connect these two different values of $P^{z}$. Note that for our purposes any mismatch in the multiplicative constant in $\zeta$ and $\tilde{\zeta}$, the analogs of $e^{-2 y_{n}}$ in Eq. (5), can be compensated by a change to $g_{q}^{S}$, so we can take these $e^{-2 y_{n}}$ constants to be 1 .

If a definition of $\tilde{\Delta}_{S}^{q}$ can be found which is calculable on the lattice and matches the IR physics of $\Delta_{S}^{q}$, then $g_{q}^{S}$ would 
become independent of $b_{T}$ and calculable in perturbation theory. In this case Eq. (24) would become a true matching relation between the quasi-TMDPDF and TMDPDF. For our analysis here we will not need to assume such an operator definition of $\tilde{\Delta}_{S}^{q}$ exists.

Note that our result in Eq. (24) differs somewhat from the analog in Ref. [10]. There the $P^{z}$ of the quasi-TMDPDF and TMDPDF were taken to be equal, so the exponential term involving $\gamma_{\zeta}^{q}$ does not appear. Also they interpreted the $b_{T}$ dependence of their $g_{q}^{S}\left(b_{T}, \mu\right)$ to be short distance without considering its nonperturbative nature for $b_{T} \sim \Lambda_{\mathrm{QCD}}^{-1}$, thus (incorrectly) concluding that Eq. (24) with a nontrivial $g_{q}^{S}\left(b_{T}, \mu\right)$ could still be interpreted as a matching equation.

\section{B. Explicit relation at one loop}

One-loop calculations comparing the quark nonsinglet quasi-TMDPDF and TMDPDF in the $\overline{\mathrm{MS}}$ scheme have been carried out in Refs. $[10,11]$ in the limit of large $P^{z}$ and $L$. Both employ a spatial quasi soft matrix element $\tilde{\Delta}_{S}^{q}$ obtained by replacing $n \rightarrow \hat{z}$ and $\bar{n} \rightarrow-\hat{z}$ in Eq. (14). Reference [11] separately calculates $\tilde{B}_{q}$ and $\tilde{\Delta}_{S}^{q}$, allowing one to easily verify the structure of Eq. (24). One obtains

$$
\begin{aligned}
C_{\mathrm{ns}}^{\mathrm{TMD}}\left(\mu, x P^{z}\right) & =1+\frac{\alpha_{s} C_{F}}{4 \pi} C^{(1)}\left(\mu, x P^{z}\right)+\mathcal{O}\left(\alpha_{s}^{2}\right), \\
C^{(1)}\left(\mu, x P^{z}\right) & =-\ln ^{2} \frac{\left(2 x P^{z}\right)^{2}}{\mu^{2}}+2 \ln \frac{\left(2 x P^{z}\right)^{2}}{\mu^{2}}-4+\frac{\pi^{2}}{6}, \\
g_{q}^{S}\left(b_{T}, \mu\right) & =1+\frac{\alpha_{s} C_{F}}{2 \pi} \ln \frac{b_{T}^{2} \mu^{2}}{4 e^{-2 \gamma_{E}}}+\mathcal{O}\left(\alpha_{s}^{2}\right) .
\end{aligned}
$$

The result of Ref. [10] corresponds to combining Eqs. (25) and (26) as in Eq. (24), and agrees with Ref. [11] up the factor $\pi^{2} / 6$ due to a different definition of the $\overline{\mathrm{MS}}$ scheme. We and Ref. [11] use $\mu_{\overline{\mathrm{MS}}}^{2}=4 \pi e^{-\gamma_{E}} \mu_{\mathrm{MS}}^{2}$, whereas Ref. [10] uses $\mu_{\overline{\mathrm{MS}}}^{2 \epsilon}=\left(4 \pi \mu_{\mathrm{MS}}^{2}\right)^{\epsilon} / \Gamma(1-\epsilon)$.

\section{EXTRACTING $\gamma_{\zeta}$ ON LATTICE}

The relation between the quasi-TMDPDF and TMDPDF in Eq. (24) allows us to extract $\gamma_{\zeta}^{q}\left(\mu, b_{T}\right)$ using lattice QCD. By considering the ratio of two copies of Eq. (24) with different hadron momenta $P_{1}^{z} \neq P_{2}^{z}$ in the quasi- TMDPDF and the same $\zeta$ in the TMDPDFs, the TMDPDFs and unknown soft contribution $g_{q}^{S}$ cancel out, yielding our main result

$$
\begin{aligned}
\gamma_{\zeta}^{q}\left(\mu, b_{T}\right) & =\frac{1}{\ln \left(P_{1}^{z} / P_{2}^{z}\right)} \ln \frac{C_{\mathrm{ns}}^{\mathrm{TMD}}\left(\mu, x P_{2}^{z}\right) \tilde{f}_{\mathrm{ns}}^{\mathrm{TMD}}\left(x, \vec{b}_{T}, \mu, P_{1}^{z}\right)}{C_{\mathrm{ns}}^{\mathrm{TMD}}\left(\mu, x P_{1}^{z}\right) \tilde{f}_{\mathrm{ns}}^{\mathrm{TMD}}\left(x, \vec{b}_{T}, \mu, P_{2}^{z}\right)} \\
& =\frac{1}{\ln \left(P_{1}^{z} / P_{2}^{z}\right)} \ln \frac{C_{\mathrm{ns}}^{\mathrm{TMD}}\left(\mu, x P_{2}^{z}\right) \int \mathrm{d} b^{z} e^{i b^{z} x P_{1}^{z}} \tilde{Z}^{\prime}\left(b^{z}, \mu, \tilde{\mu}\right) \tilde{Z}_{\mathrm{uv}}\left(b^{z}, \tilde{\mu}, a\right) \tilde{B}_{\mathrm{ns}}\left(b^{z}, \vec{b}_{T}, a, L, P_{1}^{z}\right)}{C_{\mathrm{ns}}^{\mathrm{TMD}}\left(\mu, x P_{1}^{z}\right) \int \mathrm{d} b^{z} e^{i b^{z} x P_{2}^{z}} \tilde{Z}^{\prime}\left(b^{z}, \mu, \tilde{\mu}\right) \tilde{Z}_{\mathrm{uv}}\left(b^{z}, \tilde{\mu}, a\right) \tilde{B}_{\mathrm{ns}}\left(b^{z}, \vec{b}_{T}, a, L, P_{2}^{z}\right)}
\end{aligned}
$$

The first line of Eq. (27) employs the quasi-TMDPDFs from Eqs. (22) and (23), while in the second line we have explicitly canceled out the soft factors $\Delta_{S}^{q}\left(b_{T}, a, L\right)$ to express the result entirely in terms of quasi beam function and renormalization factors. In the second line the divergences from $L \rightarrow \infty$ cancel in the ratio.

It is important to note that $\gamma_{\zeta}^{q}$ is independent of the choice for $x, P_{1}^{z}$ and $P_{2}^{z}$ on the right-hand side of Eq. (27), and any residual dependence on these can thus be used to study systematic uncertainties. Due to the universality of $\gamma_{\zeta}^{q}$, Eq. (27) can be evaluated with any hadron state (such as a pion).

It is currently not clear if the gluon anomalous dimension $\gamma_{\zeta}^{g}\left(\mu, b_{T}\right)$ can be obtained in the same manner. The concern is that in the analog of Eq. (23) the gluon could mix with the singlet quark, making the cancellation of soft factors problematic since $\tilde{\Delta}_{S}^{q} \neq \tilde{\Delta}_{S}^{g}$. Also $\gamma_{\zeta}^{g}\left(\mu, b_{T}\right)$ cannot be obtained from $\gamma_{\zeta}^{q}\left(\mu, b_{T}\right)$ with Casimir scaling, which is violated at $\mathcal{O}\left(\alpha_{s}^{4}\right)$ for $\Gamma_{\text {cusp }}^{i}$ [72-74] and thus does not hold for $\gamma_{\zeta}^{i}\left(\mu, b_{T}\right)$ nonperturbatively.

\section{A. Illustration at one loop}

For illustration, we explicitly show that we recover the correct Collins-Soper kernel at one loop. This requires the ratio of the NLO coefficient Eq. (25),

$$
\begin{aligned}
\frac{C_{\mathrm{ns}}^{\mathrm{TMD}}\left(\mu, x P_{2}^{z}\right)}{C_{\mathrm{ns}}^{\mathrm{TMD}}\left(\mu, x P_{1}^{z}\right)}= & 1+\frac{\alpha_{s} C_{F}}{\pi} \ln \frac{P_{1}^{z}}{P_{2}^{z}}\left(\ln \frac{4 x^{2} P_{1}^{z} P_{2}^{z}}{\mu^{2}}-1\right) \\
& +\mathcal{O}\left(\alpha_{s}^{2}\right),
\end{aligned}
$$

and likewise the ratio of the perturbative quasi-TMDPDFs, calculated with on-shell quark states,

$$
\begin{aligned}
\frac{\tilde{f}_{\mathrm{ns}}^{\mathrm{TMD}}\left(x, \vec{b}_{T}, \mu, P_{1}^{z}\right)}{\tilde{f}_{\mathrm{ns}}^{\mathrm{TMD}}\left(x, \vec{b}_{T}, \mu, P_{2}^{z}\right)}= & 1+\frac{\alpha_{s} C_{F}}{\pi} \ln \frac{P_{1}^{z}}{P_{2}^{z}}\left(-\ln \frac{x^{2} P_{1}^{z} P_{2}^{z} b_{T}^{2}}{e^{-2 \gamma_{E}}}+1\right) \\
& +\mathcal{O}\left(\alpha_{s}^{2}\right),
\end{aligned}
$$

which can be obtained from the results given in Appendix. Inserting Eqs. (28) and (29) into Eq. (27), we obtain 


$$
\begin{aligned}
\gamma_{\zeta}^{q}\left(\mu, b_{T}\right) & =\frac{1}{\ln \frac{P_{1}^{2}}{P_{2}^{z}}} \ln \left[1-\frac{\alpha_{s} C_{F}}{\pi} \ln \frac{P_{1}^{z}}{P_{2}^{z}} \ln \frac{b_{T}^{2} \mu^{2}}{4 e^{-2 \gamma_{E}}}+\mathcal{O}\left(\alpha_{s}^{2}\right)\right] \\
& =-\frac{\alpha_{s} C_{F}}{\pi} \ln \frac{b_{T}^{2} \mu^{2}}{4 e^{-2 \gamma_{E}}}+\mathcal{O}\left(\alpha_{s}^{2}\right),
\end{aligned}
$$

which is exactly the one-loop anomalous dimension.

\section{CONCLUSIONS AND OUTLOOK}

In this paper, we have proposed a novel approach to determine from lattice QCD the nonperturbative anomalous dimension $\gamma_{\zeta}^{q}\left(\mu, b_{T}\right)$ governing the Collins-Soper evolution of quark TMDPDFs, given in Eq. (27). It involves matrix elements of an equal-time operator with boosted hadron states (referred to as quasi beam functions), UV renormalization, and a perturbative short distance kernel $C_{\mathrm{ns}}^{\mathrm{TMD}}$. These are taken in a ratio with different hadron momentum, such that soft contributions cancel out. The nonperturbative contribution to $\gamma_{\zeta}^{q}\left(\mu, b_{T}\right)$ is required in order to evolve TMDPDFs, determined at some initial scales from experiment (or perhaps in the future a separate lattice calculation), to the scales appearing in other phenomenological applications.

So far the coefficient $C_{\mathrm{ns}}^{\mathrm{TMD}}$ is known up to one-loop order for matching an $\overline{\mathrm{MS}}$ quasi-TMDPDF to the $\overline{\mathrm{MS}}$ TMDPDF. To make use of this result for lattice calculations using nonperturbative renormalization schemes would require explicit computations of the corresponding scheme conversion factor $\tilde{Z}^{\prime}$ in Eq. (23). Related examples of nonperturbative renormalization are Refs. [1,2] for straightWilson line quasi-TMDs, Refs. [59,66] for application of the RI/MOM scheme to the quasi-PDF, and Ref. [67] for the gradient flow method. First lattice studies of the equaltime TMD quasi beam function in Eq. (20) have been carried out in Refs. [3-6]. There, ratios of this quasi beam function with $b^{z}=0$ and the same $P^{z}$ were considered, in which case renormalization factors cancel. Our method to determine $\gamma_{\zeta}^{q}$ instead requires to Fourier transform the renormalized results from $b^{z}$ into $x$ space, and consider a ratio with two different $P^{z}$ values in order to expose the Collins-Soper kernel.

A nonperturbative result for $\gamma_{\zeta}^{q}\left(\mu, b_{T}\right)$ could also have interesting applications for perturbative $b_{T}^{-1} \sim q_{T} \gg \Lambda_{\mathrm{QCD}}$.
Here it is known both from factorization and renormalon analyses [75,76] that the perturbative series for $\gamma_{\zeta}^{q}\left(\mu, b_{T}\right)$ has $\sim b_{T}^{2} \Lambda_{\mathrm{QCD}}^{2}$ power corrections, so one could attempt to obtain the coefficient of this $b_{T}^{2}$ power correction from the small $b_{T}$ limit of a nonperturbative lattice QCD result. In practice this may be difficult due to the need for a $1 /\left(b_{T} P^{z}\right)$ expansion in the relation between the quasi-TMDPDF and TMDPDF in Eq. (24).

A possible modification to our method is to consider a boosted quark state rather than a hadron state, which could have computational advantages. (We thank Will Detmold for this suggestion.) Since one can only simulate off-shell gauge-fixed quark states with Euclidean momentum $p_{E}^{2} \geq$ $\left(p^{z}\right)^{2}$ in lattice $\mathrm{QCD}$, this would require a reconsideration of various ingredients in the proposal made here. It would be interesting to pursue this in the future.

\section{ACKNOWLEDGMENTS}

We thank John Collins, Will Detmold, Markus Diehl, Michael Engelhardt and Phiala Shanahan for helpful discussions or comments. This work was supported by the U.S. Department of Energy, Office of Nuclear Physics, from DE-SC0011090 and within the framework of the TMD Topical Collaboration. I. W. S. was also supported in part by the Simons Foundation through the Investigator Grant No. 327942. M. A. E. was also supported in part by the Alexander von Humboldt Foundation through a Feodor Lynen Research Fellowship.

\section{APPENDIX: (QUASI)-TMDPDF AT NLO}

Here we summarize explicit results for the bare TMDPDF $f_{q}^{\mathrm{TMD}}\left(x, \vec{b}_{T}, \epsilon, \zeta\right)$ and quasi-TMDPDF $\tilde{f}_{q}^{\mathrm{TMD}}\left(x, \vec{b}_{T}, \epsilon, P^{z}\right)$ at one loop. These are obtained by evaluating the operators Eqs. (13) and (20) in an external quark state of lightlike momentum $p^{\mu}=\left(x P^{z}, 0,0, x P^{z}\right)$, and combining them with the appropriate soft or quasi soft function as in Eqs. (4) and (22) without performing the UV renormalization. In both cases, we use pure dimensional regularization to regulate both UV and IR divergences, and defined the $\overline{\mathrm{MS}}$ renormalization scale as $\mu_{\overline{\mathrm{MS}}}^{2}=4 \pi e^{-\gamma_{E}} \mu_{\mathrm{MS}}^{2}$.

The result for the physical TMDPDF is given by

$$
\begin{aligned}
f_{q}^{\mathrm{TMD}}\left(x, \vec{b}_{T}, \epsilon, \zeta\right)= & \delta(1-x)+\frac{\alpha_{s} C_{F}}{2 \pi}\left[-\left(\frac{1}{\epsilon_{\mathrm{IR}}}+L_{b}\right) P_{q q}(x)+(1-x)\right]_{+}^{1} \Theta(1-x) \Theta(x) \\
& +\frac{\alpha_{s} C_{F}}{2 \pi} \delta(1-x)\left[\frac{1}{\epsilon_{\mathrm{UV}}^{2}}+\left(\frac{1}{\epsilon_{\mathrm{UV}}}+L_{b}\right)\left(\frac{3}{2}+\ln \frac{\mu^{2}}{\zeta}\right)-\frac{1}{2} L_{b}^{2}+\frac{1}{2}-\frac{\pi^{2}}{12}\right]+\mathcal{O}\left(\alpha_{s}^{2}\right) .
\end{aligned}
$$

This result is obtained in the rapidity regulators of Refs. [15,26,29,32,35,37,44], which only differ by the precise definition of $\zeta$. For a more detailed comparison of results in the literature see Ref. [11]. 
For the quasi-TMDPDF, we take the physical limits $P^{z} \gg b_{T}^{-1}, L \rightarrow \infty$, and use $\tilde{\Delta}_{S}^{q}$ obtained by replacing $n \rightarrow \hat{z}$ and $\bar{n} \rightarrow-\hat{z}$ in Eq. (14). The result has been first calculated in Ref. [10] and was confirmed in Ref. [11],

$$
\begin{aligned}
\tilde{f}_{q}^{\mathrm{TMD}}\left(x, \vec{b}_{T}, \epsilon, P^{z}\right)= & \delta(1-x)+\frac{\alpha_{s} C_{F}}{2 \pi}\left[-\left(\frac{1}{\epsilon_{\mathrm{IR}}}+L_{b}\right) P_{q q}(x)+(1-x)\right]_{+}^{1} \Theta(1-x) \Theta(x) \\
& +\frac{\alpha_{s} C_{F}}{2 \pi} \delta(1-x)\left[\frac{3}{2} \frac{1}{\epsilon_{\mathrm{UV}}}-\frac{1}{2} L_{P^{z}}^{2}-L_{P^{z}}-\frac{3}{2}-\frac{1}{2} L_{b}^{2}+\frac{5}{2} L_{b}+L_{b} L_{P^{z}}\right]+\mathcal{O}\left(\alpha_{s}^{2}\right)
\end{aligned}
$$

In Eqs. (A1) and (A2), $P_{q q}(x)=\left[\left(1+x^{2}\right) /(1-x)\right]$ is the quark-quark splitting function, and $[f(x)]_{+}^{1}$ denotes the usual plus distribution such that $\int_{0}^{1} \mathrm{~d} x[f(x)]_{+}^{1}=0$. We also defined

$$
L_{b}=\ln \frac{\mu^{2} b_{T}^{2}}{4 e^{-2 \gamma_{E}}}, \quad L_{P^{z}}=\ln \frac{\mu^{2}}{\left(2 x P^{z}\right)^{2}},
$$

and made the origin of $1 / \epsilon$ poles as either IR or UV divergence explicit.
The UV renormalization factors at NLO in the $\overline{\mathrm{MS}}$ scheme are given by

$$
\begin{aligned}
Z_{\mathrm{uv}}(\mu, \epsilon, \zeta) & =1-\frac{\alpha_{s} C_{F}}{2 \pi}\left[\frac{1}{\epsilon^{2}}+\frac{1}{\epsilon}\left(\frac{3}{2}+\ln \frac{\mu^{2}}{\zeta}\right)\right]+\mathcal{O}\left(\alpha_{s}^{2}\right), \\
\tilde{Z}_{\mathrm{uv}}(\mu, \epsilon) & =1-\frac{\alpha_{s} C_{F}}{2 \pi} \frac{3}{2 \epsilon}+\mathcal{O}\left(\alpha_{s}^{2}\right) .
\end{aligned}
$$

The difference of Eqs. (A1) and (A2) after UV renormalization yields the one-loop kernel in Eqs. (25) and (26).
[1] P. Hägler, B. U. Musch, J. W. Negele, and A. Schäfer, Europhys. Lett. 88, 61001 (2009).

[2] B. U. Musch, P. Hägler, J. W. Negele, and A. Schäfer, Phys. Rev. D 83, 094507 (2011).

[3] B. U. Musch, P. Hägler, M. Engelhardt, J. W. Negele, and A. Schäfer, Phys. Rev. D 85, 094510 (2012).

[4] M. Engelhardt, P. Hägler, B. Musch, J. Negele, and A. Schäfer, Phys. Rev. D 93, 054501 (2016).

[5] B. Yoon, T. Bhattacharya, M. Engelhardt, J. Green, R. Gupta, P. Hägler, B. Musch, J. Negele, A. Pochinsky, and S. Syritsyn, in Proceedings, 33rd International Symposium on Lattice Field Theory (Lattice 2015): Kobe, Japan, 2015 (SISSA, 2015) [arXiv:1601.05717].

[6] B. Yoon, M. Engelhardt, R. Gupta, T. Bhattacharya, J. R. Green, B. U. Musch, J. W. Negele, A. V. Pochinsky, A. Schäfer, and S. N. Syritsyn, Phys. Rev. D 96, 094508 (2017).

[7] X. Ji, Phys. Rev. Lett. 110, 262002 (2013).

[8] X. Ji, Sci. China Phys. Mech. Astron. 57, 1407 (2014).

[9] X. Ji, P. Sun, X. Xiong, and F. Yuan, Phys. Rev. D 91, 074009 (2015).

[10] X. Ji, L.-C. Jin, F. Yuan, J.-H. Zhang, and Y. Zhao, arXiv:1801.05930.

[11] M. A. Ebert, I. W. Stewart, and Y. Zhao, arXiv:1901.03685.

[12] J. C. Collins and D. E. Soper, Nucl. Phys. B193, 381 (1981); B213, 545(E) (1983).

[13] J. C. Collins and D. E. Soper, Nucl. Phys. B197, 446 (1982).

[14] J. C. Collins, D. E. Soper, and G. F. Sterman, Nucl. Phys. B250, 199 (1985).
[15] J. Collins, Foundations of Perturbative QCD, Cambridge Monographs on Particle Physics, Nuclear Physics, and Cosmology (Cambridge University Press, New York, 2011).

[16] A. Bacchetta, F. Delcarro, C. Pisano, M. Radici, and A. Signori, J. High Energy Phys. 06 (2017) 081.

[17] I. Scimemi and A. Vladimirov, Eur. Phys. J. C 78, 89 (2018).

[18] J. C. Collins, D. E. Soper, and G. F. Sterman, Nucl. Phys. B261, 104 (1985).

[19] J. C. Collins, D. E. Soper, and G. F. Sterman, Nucl. Phys. B308, 833 (1988).

[20] J. C. Collins, D. E. Soper, and G. F. Sterman, Adv. Ser. Dir. High Energy Phys. 5, 1 (1989).

[21] M. Diehl, J. R. Gaunt, D. Ostermeier, P. Plöß1, and A. Schäfer, J. High Energy Phys. 01 (2016) 076.

[22] C. W. Bauer, S. Fleming, and M. E. Luke, Phys. Rev. D 63, 014006 (2000).

[23] C. W. Bauer, S. Fleming, D. Pirjol, and I. W. Stewart, Phys. Rev. D 63, 114020 (2001).

[24] C. W. Bauer and I. W. Stewart, Phys. Lett. B 516, 134 (2001).

[25] C. W. Bauer, D. Pirjol, and I. W. Stewart, Phys. Rev. D 65, 054022 (2002).

[26] T. Becher and M. Neubert, Eur. Phys. J. C 71, 1665 (2011).

[27] T. Becher, M. Neubert, and D. Wilhelm, J. High Energy Phys. 02 (2012) 124.

[28] T. Becher, M. Neubert, and D. Wilhelm, J. High Energy Phys. 05 (2013) 110.

[29] M. G. Echevarria, A. Idilbi, and I. Scimemi, J. High Energy Phys. 07 (2012) 002. 
[30] M. G. Echevarría, A. Idilbi, and I. Scimemi, Phys. Lett. B 726, 795 (2013).

[31] M. G. Echevarria, A. Idilbi, and I. Scimemi, Phys. Rev. D 90, 014003 (2014).

[32] J.-Y. Chiu, A. Jain, D. Neill, and I. Z. Rothstein, J. High Energy Phys. 05 (2012) 084.

[33] J. Collins and T. C. Rogers, Phys. Rev. D 96, 054011 (2017).

[34] S. Catani, D. de Florian, and M. Grazzini, Nucl. Phys. B596, 299 (2001).

[35] T. Becher and G. Bell, Phys. Lett. B 713, 41 (2012).

[36] T. Gehrmann, T. Lubbert, and L. L. Yang, Phys. Rev. Lett. 109, 242003 (2012).

[37] Y. Li, D. Neill, and H. X. Zhu, arXiv:1604.00392.

[38] M. G. Echevarria, I. Scimemi, and A. Vladimirov, J. High Energy Phys. 09 (2016) 004.

[39] X.-d. Ji, J.-p. Ma, and F. Yuan, Phys. Rev. D 71, 034005 (2005).

[40] A. Prokudin, P. Sun, and F. Yuan, Phys. Lett. B 750, 533 (2015).

[41] A. V. Radyushkin, Phys. Rev. D 96, 034025 (2017).

[42] J. C. Collins and F. V. Tkachov, Phys. Lett. B 294, 403 (1992).

[43] J. Collins, Proc. Sci. LC2008 (2008) 028.

[44] J.-y. Chiu, A. Jain, D. Neill, and I. Z. Rothstein, Phys. Rev. Lett. 108, 151601 (2012).

[45] M. Beneke and T. Feldmann, Nucl. Phys. B685, 249 (2004).

[46] J.-y. Chiu, F. Golf, R. Kelley, and A. V. Manohar, Phys. Rev. Lett. 100, 021802 (2008).

[47] J.-y. Chiu, A. Fuhrer, A. H. Hoang, R. Kelley, and A. V. Manohar, Phys. Rev. D 79, 053007 (2009).

[48] I. W. Stewart, F. J. Tackmann, and W. J. Waalewijn, Phys. Rev. D 81, 094035 (2010).

[49] M. G. A. Buffing, M. Diehl, and T. Kasemets, J. High Energy Phys. 01 (2018) 044.

[50] A. V. Manohar and I. W. Stewart, Phys. Rev. D 76, 074002 (2007).

[51] Y. Li and H. X. Zhu, Phys. Rev. Lett. 118, 022004 (2017).

[52] M. A. Ebert and F. J. Tackmann, J. High Energy Phys. 02 (2017) 110 .

[53] I. Scimemi and A. Vladimirov, J. High Energy Phys. 08 (2018) 003.
[54] P. Pietrulewicz, D. Samitz, A. Spiering, and F. J. Tackmann, J. High Energy Phys. 08 (2017) 114.

[55] A. A. Vladimirov, Phys. Rev. Lett. 118, 062001 (2017).

[56] Y.-Q. Ma and J.-W. Qiu, Phys. Rev. D 98, 074021 (2018).

[57] Y.-Q. Ma and J.-W. Qiu, Phys. Rev. Lett. 120, 022003 (2018).

[58] T. Izubuchi, X. Ji, L. Jin, I. W. Stewart, and Y. Zhao, Phys. Rev. D 98, 056004 (2018).

[59] M. Constantinou and H. Panagopoulos, Phys. Rev. D 96, 054506 (2017).

[60] J. Green, K. Jansen, and F. Steffens, Phys. Rev. Lett. 121, 022004 (2018).

[61] J.-W. Chen, T. Ishikawa, L. Jin, H.-W. Lin, Y.-B. Yang, J.-H. Zhang, and Y. Zhao, arXiv:1710.01089.

[62] X.-d. Ji and F. Yuan, Phys. Lett. B 543, 66 (2002).

[63] A. V. Belitsky, X. Ji, and F. Yuan, Nucl. Phys. B656, 165 (2003).

[64] A. Idilbi and I. Scimemi, Phys. Lett. B 695, 463 (2011).

[65] M. Garcia-Echevarria, A. Idilbi, and I. Scimemi, Phys. Rev. D 84, 011502 (2011).

[66] I. W. Stewart and Y. Zhao, Phys. Rev. D 97, 054512 (2018).

[67] C. Monahan and K. Orginos, J. High Energy Phys. 03 (2017) 116

[68] X. Ji, J.-H. Zhang, and Y. Zhao, Phys. Rev. Lett. 120, 112001 (2018).

[69] T. Ishikawa, Y.-Q. Ma, J.-W. Qiu, and S. Yoshida, Phys. Rev. D 96, 094019 (2017).

[70] J.-W. Chen, S. D. Cohen, X. Ji, H.-W. Lin, and J.-H. Zhang, Nucl. Phys. B911, 246 (2016).

[71] X. Xiong, X. Ji, J.-H. Zhang, and Y. Zhao, Phys. Rev. D 90, 014051 (2014).

[72] R. H. Boels, T. Huber, and G. Yang, Phys. Rev. Lett. 119, 201601 (2017).

[73] S. Moch, B. Ruijl, T. Ueda, J. A. M. Vermaseren, and A. Vogt, J. High Energy Phys. 10 (2017) 041.

[74] A. Grozin, J. Henn, and M. Stahlhofen, J. High Energy Phys. 10 (2017) 052.

[75] T. Becher and G. Bell, Phys. Rev. Lett. 112, 182002 (2014).

[76] I. Scimemi and A. Vladimirov, J. High Energy Phys. 03 (2017) 002. 